\title{
High-Performance Algorithms for Quantum Systems Evolution
}

\author{
Alexander V. Bogdanov, Ashot S. Gevorkyan, and Elena N. Stankova \\ Institute for High Performance Computing and Data Bases, \\ Fontanka, 118, 198005, St-Petersburg, Russia, \\ bogdanov@hm.csa.ru, ashot@fn.csa.ru, lena@fn.csa.ru
}

\begin{abstract}
We discuss some new approach for derivation of computational algorithms for evolution of quantum systems. The main idea of the algorithm is to make in functional or path representation of quantum observable the transformation of variables, that makes the phase of path integral to be quadric functional. Thus the new representation for observable is reduced to standard multidimensional integral and the solution, for every point in coordinate space of that integral, of the first order partial differential equation system. That problem, although still difficult, can be very effectively parallelyzed. The approach is illustrated with the help of important example - the scattering in molecular system with elementary chemical reactions. The use of proposed approach show substantial speed-up over the standard algorithms and seems to be more effective with the increase of the size of the problem.
\end{abstract}

\section{Introduction}

Even with increase of the power of computers, used for quantum collision problem analysis nowadays, we do not feel the drastic increase of computational possibilities, especially when the number of nodes in computations is high. After some analysis it does not seem so strange since with the increase of number of parallel computational processes the price, which we pay for exchange of data between processes, is becoming more and more heavy load, that make the increase of number of nodes ineffective. That is why it is very important in deriving the algorithms to minimize the data exchange between computational processes. The standard algorithms for quantum scattering calculations are very ineffective for complex systems since in many realistic situations strong coupling between large amount of interacting states should be taken into account additionally to nontrivial problems with asymptotic boundary conditions. Among many candidates for alternative approach one of the most promising is the reduction of the original formulation to Feynman's path integral. One of the firsts such effective algorithms was proposed in 1991 ([1]). But due to some mathematical properties of path integrals this approach could be effectively used only for finite time interval evolution calculation. Several attempts were made to overcome those difficulties by describing the evolution in classical terms and solving large time problems by classical means. A lot of work was done in establishing rigorous 
relationship between classical and quantum tools for description of system evolution ([2]). Although formal mathematical solution of the problem was given in 1979 ([3]), and computational algorithm was proposed in 1986 (4]), only recently it was possible to realize it on large systems ([5]). The main result of $([\overrightarrow{3}])$ was the functional transformation to so called interaction coordinates, that reduces Hamiltonian function of the problem to actually responsible for described process and reduces integration interval to finite one, corresponding to interaction region. More than that it transforms asymptotical boundary conditions to standard ones and makes it possible to get the expression directly to scattering amplitude as an average over Green's functions of the problem but in mixed representation and in interaction coordinates.

$$
A\left(\boldsymbol{i}, \boldsymbol{f}^{\prime}\right)=\left\langle\boldsymbol{G}(\boldsymbol{i}, \boldsymbol{t}) \boldsymbol{G}\left(\boldsymbol{t}, \boldsymbol{f}^{\prime}\right)\right\rangle .
$$

The average is taken over the coordinate space of the problem with Green's functions determined in terms of path integrals over the phase space with the weights of the type

$$
\exp \left(-i+\int X d P+i X P-i \int H d t\right)
$$

i.e. the classical action in phase space in standard notation ([1]). Since we use Green's functions only for computation of averages (1) we can make any phase coordinates transformations of the type

$$
H(P, X) \rightarrow H\left(X, \frac{\partial F}{\partial X}\right)+\frac{\partial F}{\partial t}
$$

With $F$ being the Generator of the transformation. It is convenient to choose $F$ as a solution of certain equation (4] ) that guarantees the possibility of evaluation of path integral with the weight (2). In that case instead of computation of path integral we have to solve four times the equation for $F$, that is more convenient since it is partial differential equation of the first order $([3])$. The resulting amplitude representation is multidimensional integral:

$$
\begin{gathered}
T\left(P_{i} \rightarrow P_{f}\right)=\int d X_{0} C \delta\left(X_{0}, P_{0}\right) \exp \left\{\frac{i}{\hbar}\left(P_{f} X_{f}-P_{i} X_{i}\right)+\right. \\
\left.+\left.\frac{i}{\hbar} F_{1}\right|_{i} ^{0}+\left.\frac{i}{\hbar} F_{1}\right|_{0} ^{f}+\frac{i}{\hbar} Q_{i}\left(Y_{0}-Y_{i}\right)+\frac{i}{\hbar} Q_{f}\left(Y_{f}-Y_{0}\right)\right\} .
\end{gathered}
$$

\section{Numerical Method}

So in general case computation of scattering amplitude in our approach is reduced to computation of average, i.e. of integral over the coordinate space and solution for every coordinate of four partial differential equations of the first order for $F$. It is clear, that such formalism gives an ideal parallel algorithm, since we can do the solutions of equations for different points independently. More, 
than that, we can make the same sort of diagonal approximation as in coupled states approach and choose one average solution for all four generators $F$. This is the equivalent of so called average trajectory approximation in semiclassical approach. Schematically the algorithm of such process computation can be presented in the following way $([6])$ :

I - Lagrangian surface construction for the system. The curvilinear coordinate system, within which all the further calculations are performed, is derived in it; II - Classical trajectory problem solution. At this stage the system of ordinary non-linear differential equations of the first order is being solved numerically. The problem's parameters are collision energy $E$ and quantum numbers of initial configuration $n$. This system is being solved by one-step method of 4th-5th order of accuracy. This method is conditionally stable (by initial deviation and right part), that's why the standard automatic step decreasing method is implied to provide its stability. It's worth mentioning that initial system degenerates in certain points. To eliminate this degeneration, the standard -procedure with differentiation parameter replacement is performed.

III - The results of classical trajectory problem calculation are used for quantum calculations performing and complete wave function obtaining in its final state. At this stage, the numerical problem represents solution of an ordinary non-linear differential equation of the second order. Calculating this equation is a difficult task due to non-trivial behavior of differentiation parameter (7]). Differentiation algorithm consists of two stages: 1) construction of differentiation parameter values system using the results of classical problem calculation and 2) integration of initial differential equation on non-uniform system obtained by means of multi-step method. Choosing such integration step in a classical problem provides integration stability, while control is performed by means of step-by-step truncation error calculation. The obtained solution of differential equation is approximated in a final asymptote in a form of falling and reflected flat wave superposition;

Let's remind that calculations for steps II and III are made for specific values of collision energy $\mathrm{E}$ and oscillation quantum number of initial state. Results of these calculations allow to obtain one line of a transition matrix, which corresponds to $\boldsymbol{n}$. In order to obtain the entire transition matrix, calculations at stages II and III need to be repeated as many times as dictated by the size of transitional probability matrix. As a result the entire probability matrix is obtained. The procedure described needs to be repeated for many values of collision energy in order to enable further integration and velocity constants finding. It is clear, that most time consuming are the stages II and III and that they can be carried to large extend on independent computational systems, using one of them just to collect all the results and work out the statistics. Since from each of such computation we need only the value of the kernel of transition functional it was possible to make exchange of such information as low as possible. All the computations of the stages IV and V were carried out on MPP system Parsytec CCe-20 of IHPCDB and the individual computations for different trajectories on MPP IBM SP-2 of GMD. We found, that MPP architecture, although old-fashioned, is very 
well suited for the architecture of proposed algorithm. The parallelization was performed for the values of collision energy. Calculation of classical trajectory problem, quantum calculation and transition probability matrix calculation is performed in each of the parallel branches. Let's note that just as in the case on non-parallelized algorithm all calculations from stages II and III are performed as many times as it is dictated by the size of transition probability matrix. Due to the fact that calculation in each of the parallel branches represents a separate problem and does not interact with other branches in calculation, the effectiveness of using this parallelization algorithm vs. relatively unparallelized algorithm is nearly proportional to a number of calculation branches, i.e. to the amount of computation nodes.

\section{Numerical Example}

As a reaction on which the algorithm was tested, a well studied bimolecular reaction $\mathrm{Li}+(\mathrm{FH})(\mathrm{LiFH})^{*}(\mathrm{LiH})+\mathrm{H}$ was taken. The potential surface for this reaction was reproduced using the quantum-mechanical calculations carried out in work $([8])$. The results of testing have shown the calculation effectiveness to be nearly proportional to the number of computation nodes. We have proposed the variant of our approach for the shared memory systems. However now we have no technical possibilities to unite in large clusters systems with shared memory emulation in the regime of NUMA architecture. But this problem solution is one of the main items in the program of joint activities with GMD in the nearest years Finally we would like to stress one of the peculiarities of parallelization algorithms demonstrated - their scalability. Due to the fact that integration of transition probability matrix and rate constants calculation during stage $\mathrm{V}$ requires the values of matrix elements for many energy values, one can hardly find a supercomputer with an excessive number of computation nodes. As illustration (Fig. 1) we show first exact converging results of computation of reaction probability and properties of the system.

\section{Conclusions}

We have shown that the use of some physical considerations makes it possible to derive some new algorithms for solution of the evolution equations for physical variables like wave function. With those algorithms we can reduce the needed computer time orders of magnitude, go to substantially larger number of processor and work out approximate methods, which can be used for mass computations in technical applications. Scalability of these algorithms was used for conducting distributed computing runs on the supercomputers of GMD and SARA. Internet possibilities allowed to obtain access for most difficult part of the problem - trajectories calculations - to the far more powerful computing resources that are available in IHPCDB, and so to conduct distributed computations in the different regimes including $\mathrm{X}$ terminal regime. In the future we are planning to provide in the similar approach the visualization of the numerical 
results, data preparations and preliminary tests for remote compilation on the cluster of workstations Octane and Sun Ultra, available in IHPCDB and sending them for further processing to the ONYX visualization supercomputers situated in GMD and SARA. At the same time the proposed approach really make it possible to get on large scale problems substantial speed-ups over the standard algorithms.
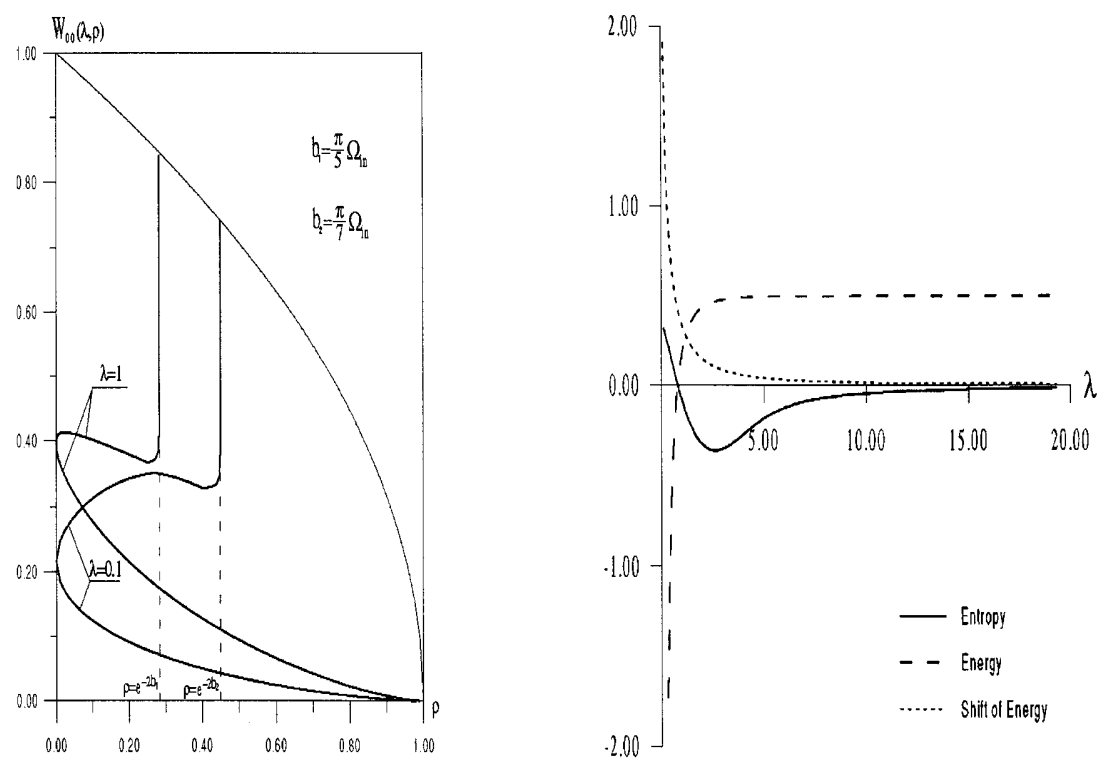

Fig. 1. The results of the first exact computation of the probability dependencies for reaction $\mathrm{Li}+(\mathrm{FH})(\mathrm{LiFH})^{*}(\mathrm{LiH})+\mathrm{H}$

\section{References}

[1] Topaler M., Makri N.: Multidimensional path integral calculations with quasidiabatic propagators: Quantum dynamics of vibrational relaxation in linear hydrocarbon chains. J.Chem.Phys. Vol. 97, 12, (1992) 9001-9015

[2] Greenberg W.R., Klein A., Zlatev I.: From Heisenberg matrix mechanics to semiclassical quantization: Theory and first applications. Phys. Rev. A Vol.54, 3 , (1996) 1820-1836.

[3] Dubrovskiy G.V., Bogdanov A.V. Chem.Phys.Lett., Vol. 62, 1 (1979) 89-94.

[4] Bogdanov A.V.: Computation of the inelastic quantum scattering amplitude via the solution of classical dynamical problem. In:Russian Journal of Technical Physics, 7 (1986) 1409-1411. 
[5] A.V. Bogdanov, A.S. Gevorkyan, A.G. Grigoryan, Stankova E.N.: Use of the Internet for Distributed Computing of Quantum Evolution. in Proccedings of 8th Int. Conference on High Performance Computing and Networking Europe (HPCN Europe '2000), Amsterdam, The Netherlands (2000)

[6] A.V. Bogdanov, A.S. Gevorkyan, A.G. Grigoryan and S.A. Matveev, Investigation of High-Performance Algorithms for Numerical Calculations of Evolution of Quantum Systems Based on Their Intrinsic Properties in Proccedings of 7th Int. Conference on High Performance Computing and Networking Europe (HPCN Europe '99), Amsterdam, The Netherlands, and April 12-14, 1999, pp.1286-1291.

[7] A.V. Bogdanov, A.S. Gevorkyan, A.G. Grigoryan, First principle calculations of quantum chaos in framework of random quantum reactive harmonic oscillator theory, in Proceedings of 6th Int. Conference on High Performance Computing and Networking Europe (HPCN Europe '98), Amsterdam, The Netherlands, April, 1998.

[8] S. Carter, J. N. Murrell, Analytical Potentials for Triatomic Molecules, Molecular Physics, v. 41, N. 3, pp. 567-581, (1980). 